(C) 2006 IEEE. Personal use of this material is permitted. Permission from IEEE must be obtained for all other uses, in any current or future media, including reprinting/republishing this material for advertising or promotional purposes, creating new collective works, for resale or redistribution to servers or lists, or reuse of any copyrighted component of this work in other works. 


\title{
Multiquadrics Collocation Method for Transient Eddy Current Problems
}

\author{
Yong Zhang, K.R. Shao, Youguang Guo, and J.D. Lavers
}

\begin{abstract}
This paper presents the multiquadrics collocation method for transient eddy current problems. Both the implicit scheme and Crank-Nicolson time matching scheme are used here for time discretization. An example on analyzing transient eddy current of a square metal column is set to prove the accuracy and affectivity of the proposed method.
\end{abstract}

Index Terms - Transient eddy current, multiquadics, initialboundary problem, meshless method

\section{INTRODUCTION}

M ESHLESS or Meshfree methods form a new class of numerical techniques of which the main objective is to overcome the limitations imposed by traditional mesh structured methods [1]. This type of methods uses a set of nodes distributed in solving domain instead of traditional elements. Meshless techniques are divided into two classes: those based on collocations and those based on weak forms. The first class is truly meshless method and do not require a mesh structure or a numerical integration procedure, it is insensitive to spatial dimension, considering only a cloud of nodes for the spatial discretization of both the solving domain and the boundary.

Radial basis function (RBF) method, as a truly meshless method for approximating the solutions of PDEs, has drawn much of the attention of many researchers in science and engineering [2]. In this class of truly RBF meshless methods, the multiquadrics collocation method is ranked the best based on its high accuracy, ease of implementation, good visual aspect, low execution time and storage requirements [3]. Multiquadrics are only dependent on spatial coordinates. This characteristic imposes the use of mixed algorithms to treat time-dependent problems. From Newton iteration to the forward difference scheme, it is possible to combine a wide variety of time-dependent solvers with the basic RBF spatial treatment, used for examples in hyperbolic equation [4].

In this paper, multiqudrics collocation method is applied to transient eddy current problems, and both the implicit scheme and Crank-Nicolson time matching scheme are used here for time discretization. An example on analyzing transient eddy current of a square metal column is set here to illustrate the accuracy and affectivity of the proposed method.

This work was supported by National Natural Science Foundation of China under Grant No.50477045 and Specialized Research Funds for the Doctoral Program of High Education under Grant 20030478063.

Yong Zhang and K.R. Shao are with the College of Electrical \& Electronic Engineering, Huazhong University of Science and Technology, Wuhan 430074 P.R. China. (E-mail: krshao@hust.edu.cn)

Y. Guo is with the Faculty of Engineering, University of Technology, Sydney, NSW 2007, Australia (e-mail: youguang@eng.uts.edu.au).

J.D. Lavers is with the Department of Electrical \& Computer Engineering, University of Toronto, Toronto, M5S 3G4, Canada.

\section{Multiquadrics COllocation Method}

The multiquadrics collocation method belongs to RBF type methods. Consider a set of nodes $x_{1}, x_{2}, \cdots, x_{N} \in \mathbf{R}^{n}$. The $\mathrm{RBF}$ centered at $\mathbf{x}_{j}$ are defined as

$$
\varphi_{j}(\mathbf{x}) \equiv \varphi\left(\left\|\mathbf{x}-\mathbf{x}_{j}\right\|, c\right) \in \mathbf{R}^{n}, j=1,2, \cdots, N
$$

where $\left\|\mathbf{x}-\mathbf{x}_{j}\right\|$ is the Euclidian norm. The RBF-MQ is

$$
\varphi_{j}(\mathbf{x})=\left(\left\|\mathbf{x}-\mathbf{x}_{j}\right\|+c^{2}\right)^{1 / 2}
$$

where $c$ is a shape parameter. Let $\Omega \subset \mathbf{R}^{n}$, we consider a linear elliptic boundary value problem of the form

$$
\begin{cases}\operatorname{Lu}(x)=f(x) & \text { in } \Omega \subset \mathbf{R}^{n} \\ B u(x)=g(x) & \text { on } \partial \Omega\end{cases}
$$

where $\Omega$ is a bounded domain with the boundary $\partial \Omega, L$ is a linear elliptic partial differential operator, and $B$ is a boundary operator. We use $N$ distinct nodes in and on the boundary of $\Omega$, of which $\left\{\mathbf{x}_{j}, j=1, \cdots N_{I}\right\}$ are interior nodes, $\left\{\mathbf{x}_{j}, j=N_{I}+1, \cdots N\right\}$ are boundary points. We look for the approximate solution $u_{h}(x)$ to (3) in the form

$$
u_{h}(x)=\sum_{j=1}^{N} a_{j} \varphi\left(\left\|\mathbf{x}-\mathbf{x}_{j}\right\|, c\right)=\sum_{j=1}^{N} a_{j} \varphi_{j}(\mathbf{x})
$$

where $\mathbf{a}=\left[a_{1}, \cdots, a_{N}\right]$ are the unknown coefficients to be determined. Substituting $u_{h}(x)$ into (3) and using collocation at the $N$ nodes, we can get the finite dimensional problem

$$
\begin{aligned}
& \sum_{j=1}^{N} a_{j} L\left(\varphi_{j}\left(\mathbf{x}_{i}\right)\right)=f\left(\mathbf{x}_{i}\right), i=1, \cdots N_{I} \\
& \sum_{j=1}^{N} a_{j} B\left(\varphi_{j}\left(\mathbf{x}_{i}\right)\right)=g\left(\mathbf{x}_{i}\right), i=N_{I}+1, \cdots N
\end{aligned}
$$

This corresponds to the system of equations with a coefficient matrix, its matrix form and solution are

$$
\begin{gathered}
{\left[\begin{array}{l}
\mathbf{L}(\varphi) \\
\mathbf{B}(\varphi)
\end{array}\right][\mathbf{a}]=\left[\begin{array}{l}
\mathbf{f} \\
\mathbf{g}
\end{array}\right]} \\
{[\mathbf{a}]=\left[\begin{array}{l}
\mathbf{L}(\varphi) \\
\mathbf{B}(\varphi)
\end{array}\right]^{-1}\left[\begin{array}{l}
\mathbf{f} \\
\mathbf{g}
\end{array}\right]}
\end{gathered}
$$

In (6), we have

$$
\begin{aligned}
\mathbf{L}(\varphi) & =\left[\begin{array}{ccc}
L\left(\varphi_{1}\left(\mathbf{x}_{1}\right)\right) & \cdots & L\left(\varphi_{N}\left(\mathbf{x}_{1}\right)\right) \\
\vdots & \vdots & \vdots \\
L\left(\varphi_{1}\left(\mathbf{x}_{N I}\right)\right) & \cdots & L\left(\varphi_{N}\left(\mathbf{x}_{N I}\right)\right)
\end{array}\right] \\
\mathbf{B}(\varphi) & =\left[\begin{array}{ccc}
B\left(\varphi_{1}\left(\mathbf{x}_{N I+1}\right)\right) & \cdots & B\left(\varphi_{N}\left(\mathbf{x}_{N I+1}\right)\right) \\
\vdots & \vdots & \vdots \\
B\left(\varphi_{1}\left(\mathbf{x}_{N}\right)\right) & \cdots & B\left(\varphi_{N}\left(\mathbf{x}_{N}\right)\right)
\end{array}\right]
\end{aligned}
$$


The use of globally supported RBF as multiquadrics for large problems can bring problems due to its full populated matrices. Domain decomposition methods and localization of the basis functions can be used to solve such drawback [5]. The present model does not issue such methods, for the problems dealt with in the paper the number of nodes to provide good quality solutions is usually small. For large applications with complex geometry or vast number of nodes, such refined methods are certainly needed.

\section{TRANSIENT EDDY CURRENT PROBLEM}

The full set of equations for low-frequency electromagnetic field can be now written as

$$
\begin{gathered}
\nabla \times \mathbf{E}=-\frac{\partial \mathbf{B}}{\partial t}, \quad \mathbf{B}=\mu \mathbf{H} \\
\nabla \times \mathbf{H}=\mathbf{J}_{e}+\mathbf{J}_{s}, \mathbf{J}_{e}=\sigma \mathbf{E}
\end{gathered}
$$

where $\mathbf{J}_{e}$ and $\mathbf{J}_{s}$ are eddy current and source current respectively. For linear conductive medium, with Lorentz gauge, the equivalent form by using magnetic potential vector of equation (8) can be written as follows

$$
\nabla^{2} \mathbf{A}=\sigma \mu \frac{\partial \mathbf{A}}{\partial t}-\mu \mathbf{J}_{s}
$$

Therefore, for two-dimensional problems, equation (9) can be reduced to a scalar diffusion equation as follows

$$
\nabla^{2} u=\sigma \mu \frac{\partial u}{\partial t}-f
$$

Where $u$ is one of the three components of vector potential $\mathbf{A}$, $f$ is the supplied source. The initial and boundary problems for two-dimensional transient eddy current field is written as

$$
\left\{\begin{array}{cc}
\alpha \frac{\partial u}{\partial t}-L(u)=f & \text { in } \Omega \text { at } 0<t<t_{n} \\
B(u)=g & \text { on } \partial \Omega \text { at } 0 \leq t<t_{n}
\end{array}\right.
$$

where $\alpha=\sigma \mu, L=\nabla^{2}(\cdot)$ and $\Omega$ is the solver domain with boundary $\partial \Omega$. We approximate $u$ by $u_{h}(t, x)$ and assume

$$
u_{h}(t, x)=\sum_{j=1}^{N} a_{j}(t) \varphi\left(\left\|\mathbf{x}-\mathbf{x}_{j}\right\|, c\right)=\sum_{j=1}^{N} a_{j}(t) \varphi_{j}(\mathbf{x})
$$

where $\mathbf{a}(t)=\left[a_{1}(t), \cdots, a_{N}(t)\right]$ are unknown coefficients to be determined at each time step. The derivative of the approximating solution on time is

$$
\frac{\partial u_{h}(t, x)}{\partial t}=\sum_{j=1}^{N} \frac{\partial a_{j}(t)}{\partial t} \varphi_{j}(\mathbf{x})
$$

and spatial derivatives are obtained as

$$
L\left(u_{h}(t, x)\right)=\sum_{j=1}^{N} a_{j}(t) L\left(\varphi_{j}(\mathbf{x})\right)
$$

Substituting (13) and (14) into (11) and collocation at $N$ nodes, we can get the form

$$
\begin{gathered}
\sum_{j=1}^{N}\left(\alpha \frac{\partial a_{j}(t)}{\partial t} \varphi_{j}\left(\mathbf{x}_{i}\right)-a_{j}(t) L\left(\varphi_{j}\left(\mathbf{x}_{i}\right)\right)\right)=f\left(\mathbf{x}_{i}\right) \\
i=1, \cdots N_{I}
\end{gathered}
$$

$$
\sum_{j=1}^{N} a_{j}(t) B\left(\varphi_{j}\left(\mathbf{x}_{i}\right)\right)=g\left(\mathbf{x}_{i}\right), i=N_{I}+1, \cdots N
$$

which can be expressed as

$$
\begin{array}{r}
\alpha \mathbf{\Phi} \dot{\mathbf{a}}+\boldsymbol{\Phi}_{L} \mathbf{a}=\mathbf{f} \\
\boldsymbol{\Phi \mathbf { a }}=\mathbf{g}
\end{array}
$$

where $\boldsymbol{\Phi}=\varphi\left(\left\|\mathbf{x}-\mathbf{x}_{j}\right\|, c\right)$ and $\boldsymbol{\Phi}_{L}=\mathbf{L}\left(\varphi\left(\left\|\mathbf{x}-\mathbf{x}_{j}\right\|, c\right)\right)$.

For RBF-MQ in 2D Cartesian coordinate, we have

$$
\begin{gathered}
\varphi_{j}(\mathbf{x})=\sqrt{\left(x-x_{j}\right)^{2}+\left(y-y_{j}\right)^{2}+c^{2}}=\sqrt{r_{j}^{2}+c^{2}} \\
r=\sqrt{\left(x-x_{j}\right)^{2}+\left(y-y_{j}\right)^{2}} \\
\frac{\partial \varphi_{j}(\mathbf{x})}{\partial x}=\frac{x-x_{j}}{\sqrt{r_{j}^{2}+c^{2}}} \\
\frac{\partial \varphi_{j}(\mathbf{x})}{\partial y}=\frac{y-y_{j}}{\sqrt{r_{j}^{2}+c^{2}}} \\
\frac{\partial^{2} \varphi_{j}(\mathbf{x})}{\partial x^{2}}=\frac{\left(y-y_{j}\right)^{2}+c^{2}}{\left(r_{j}^{2}+c^{2}\right)^{3 / 2}} \\
\frac{\partial^{2} \varphi_{j}(\mathbf{x})}{\partial y^{2}}=\frac{\left(x-x_{j}\right)^{2}+c^{2}}{\left(r_{j}^{2}+c^{2}\right)^{3 / 2}} \\
L\left(\varphi_{j}(\mathbf{x})\right)=\frac{\partial^{2} \varphi_{j}(\mathbf{x})}{\partial x^{2}+\frac{\partial^{2} \varphi_{j}(\mathbf{x})}{\partial y^{2}}=\frac{r^{2}+2 c^{2}}{\left(r_{j}^{2}+c^{2}\right)^{3 / 2}}}
\end{gathered}
$$

In this paper, two practical difference scheme on time disretization are disscussed, they are implicit scheme and Crank-Nicolson time matching scheme respectively.

\section{A. Implicit scheme}

For the problem described by (16), we can use the following implicit scheme for a discretization of time variable

$$
\begin{aligned}
\alpha \boldsymbol{\Phi} \frac{\left(\mathbf{a}^{n+1}-\mathbf{a}^{n}\right)}{\Delta t} & =\boldsymbol{\Phi}_{L} \mathbf{a}^{n+1}+\mathbf{f} \\
\boldsymbol{\Phi a}^{n+1} & =\mathbf{g},
\end{aligned}
$$

whose iterative scheme is

$$
\begin{gathered}
\left(\frac{\alpha}{\Delta t} \mathbf{\Phi}-\boldsymbol{\Phi}_{L}\right) \mathbf{a}^{n+1}=\frac{\alpha}{\Delta t} \boldsymbol{\Phi}^{n}+\mathbf{f} \\
\boldsymbol{\Phi a}^{n+1}=\mathbf{g}
\end{gathered}
$$

Combining with (15), (19) can be expressed as

$$
\begin{gathered}
\begin{aligned}
\sum_{j=1}^{N}\left(\frac{\alpha}{\Delta t} \varphi_{j}\left(\mathbf{x}_{i}\right)-L\left(\varphi_{j}\left(\mathbf{x}_{i}\right)\right)\right) a_{j}\left(t_{n+1}\right)=\frac{\alpha}{\Delta t} \sum_{j=1}^{N} \varphi_{j}(\mathbf{x}) a_{j}\left(t_{n}\right) \\
+f\left(\mathbf{x}_{i}\right), \quad i=1, \cdots N_{I}
\end{aligned} \\
\sum_{j=1}^{N} B\left(\varphi_{j}\left(\mathbf{x}_{i}\right)\right) a_{j}\left(t_{n+1}\right)=g\left(\mathbf{x}_{i}\right), \quad i=N_{I}+1, \cdots N
\end{gathered}
$$

The algorithms for (19) in time iteration are formulated as

1. Initialize at time $t:=0$, approximate the initial condition by using (12) and then calculate the partial differential operator $\mathbf{L}\left(\varphi\left(\left\|\mathbf{x}-\mathbf{x}_{j}\right\|, c\right)\right)$.

2. Solve (19) to obtain coefficient $\boldsymbol{\alpha}$ and then compute the solution $\mathbf{u}$ by using (12) and then calculate the partial 
differential operator $\mathbf{L}\left(\varphi\left(\left\|\mathbf{x}-\mathbf{x}_{j}\right\|, c\right)\right)$ at time $t:=t$.

3. Correct the boundary values by using boundary conditions with (19.b).

4. Put time to $t:=t+\Delta t$, go to Step2 until time $t:=t_{n}$.

\section{B. Crank-Nicolson( $C-N)$ time matching scheme}

In the Crant-Nicolson formulation, the iterative scheme for time discretization becomes

$$
\begin{aligned}
\alpha \boldsymbol{\Phi} \frac{\left(\mathbf{a}^{n+1}-\mathbf{a}^{n}\right)}{\Delta t} & =\boldsymbol{\Phi}_{L} \frac{\left(\mathbf{a}^{n+1}+\mathbf{a}^{n}\right)}{2}+\mathbf{f} \\
\mathbf{\Phi a}^{n+1} & =\mathbf{g}
\end{aligned}
$$

whose iterative scheme is

$$
\begin{aligned}
\left(\frac{\alpha \boldsymbol{\Phi}}{\Delta t}-\frac{\boldsymbol{\Phi}_{L}}{2}\right) \mathbf{a}^{n+1} & =\left(\frac{\alpha \boldsymbol{\Phi}}{\Delta t}+\frac{\boldsymbol{\Phi}_{L}}{2}\right) \mathbf{a}^{n}+\mathbf{f} \\
\boldsymbol{\Phi}^{n+1} & =\mathbf{g}
\end{aligned}
$$

Combining with (15), (22) can be expressed as

$$
\begin{aligned}
& \sum_{j=1}^{N}\left(\frac{\alpha \varphi_{j}\left(\mathbf{x}_{i}\right)}{\Delta t}+\frac{L\left(\varphi_{j}\left(\mathbf{x}_{i}\right)\right)}{2}\right) a_{j}\left(t_{n}\right)=f\left(\mathbf{x}_{i}\right) \\
& +\sum_{j=1}^{N}\left(\frac{\alpha \varphi_{j}\left(\mathbf{x}_{i}\right)}{\Delta t}+\frac{L\left(\varphi_{j}\left(\mathbf{x}_{i}\right)\right)}{2}\right) a_{j}\left(t_{n}\right), \quad i=1, \cdots N_{I} \\
& \sum_{j=1}^{N} B\left(\varphi_{j}\left(\mathbf{x}_{i}\right)\right) a_{j}\left(t_{n+1}\right)=g\left(\mathbf{x}_{i}\right), \quad i=N_{I}+1, \cdots N
\end{aligned}
$$

In Crank-Nicolson time matching scheme, the remaining iterative procedure follows the same scheme as in the implicit scheme. Both the implicit scheme and the Crank-Nicolson time matching scheme are absolutely stable difference scheme, and they are both suitable for hyperbolic problems as transient eddy current analysis.

\section{NUMERICAL EXAMPLE}

In order to verify the proposed method, a metal column with infinite length is magnetized here. Its cross section of one quadrant is shown as Fig.1. The parameters of the size and the medium type are: $\mathrm{OA}=0.4 \mathrm{~m}, \mathrm{OB}=0.2 \mathrm{~m}, \mu_{r}=1000$, $\sigma=1.04 \times 10^{6} \mathrm{~S} / \mathrm{m}$. At time $t=0$, a step magnetic field $H_{0}$ with direction $-z$ is imposed on the outer surface of the metal column. There are total 153 nodes distributed in the solving domain, 105 nodes for interior and 48 nodes for boundary respectively. Points $P(0.1,0.1)$ are investigated here to compare the exact solution and the numerical one by using the proposed two schemes for time discretization. The comparative results are shown in Table 1 . As can be seen from Table 1, results obtained from the present methodology are in excellent with exact results. Fig.2 shows the eddy current distribution of the metal column at time $t=80.0 \mathrm{~s}$.

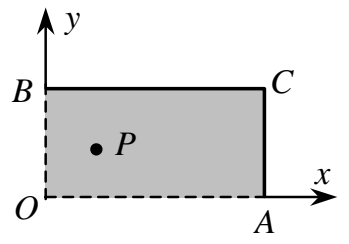

Fig.1 Cross section of one quadrant of the metal column
TABLE 1

A COMPARISON OF EXACT SOLUTION AND THE NUMERICAL ONE

\begin{tabular}{|c|c|c|c|}
\hline Time $t$ & \multirow{2}{*}{$\begin{array}{c}\text { Exact } \\
(\mathrm{s})\end{array}$} & solution & \multicolumn{2}{|c|}{ Numerical solution } \\
\cline { 3 - 4 } & Implicit scheme & C-N scheme \\
\hline 0.0 & 0.0000 & 0.0023 & -0.0018 \\
\hline 16.0 & 0.2465 & 0.2502 & 0.2474 \\
\hline 32.0 & 0.4976 & 0.4988 & 0.4986 \\
\hline 48.0 & 0.6581 & 0.6594 & 0.6591 \\
\hline 64.0 & 0.7660 & 0.7668 & 0.7666 \\
\hline 80.0 & 0.8396 & 0.8412 & 0.8409 \\
\hline 96.0 & 0.8901 & 0.8908 & 0.8896 \\
\hline 112.0 & 0.9246 & 0.9244 & 0.9252 \\
\hline 128.0 & 0.9483 & 0.9487 & 0.9491 \\
\hline 142.0 & 0.9645 & 0.9638 & 0.9642 \\
\hline 160.0 & 0.9757 & 0.9763 & 0.9752 \\
\hline
\end{tabular}

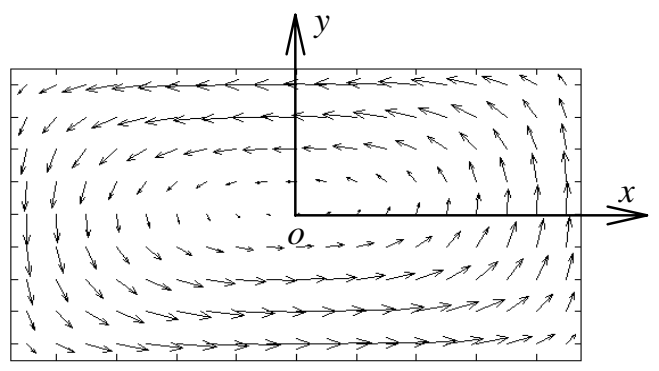

Fig.2 Eddy current distribution of the metal column at $t=80.0 \mathrm{~s}$

\section{CONCLUSIONS}

Multiquadics collocation meshless method for transient current problems is presented in this paper. This method is truly meshless method and does not require a mesh structure or a numerical integration procedure, it is insensitive to spatial dimension, considering only a cloud of nodes for the spatial discretization of both the solving domain and the boundary. Numerical example proves the present method is with high accuracy, ease of implementation, low execution time and storage requirements. With comparison to conventional FEM, the present method uses a scattered set of points instead of elements and it should be more flexible than the latter one, which also encounters difficulties in the treatment of discontinuities that might not necessarily coincide with the element boundaries. Future work is in progress to apply the method in other realistic engineering problems such as 3D transient eddy current problems and the propagating cracks in nondestructive evaluation.

\section{REFERENCES}

[1] G. R. Liu, Mesh Free Methods: Moving beyond the Finite Element Method, 1st ed. USA: CRC PRESS, 2003.

[2] C. Franke, R. Schaback, "Solving Partial Differential Equations by Collocation Using Radial Basis Functions,” Appl. Math. Comp., vol.93, no.1, pp.73-91, 1998.

[3] R. Franke, "Scattered Data Interpolation: Test of Some Method," Math. Comput., vol.48, pp.381-399, 1982.

[4] J.C. Li, Y.C. Hon, C.S. Chen, "Numerical Comparisons of Two Meshless Method Using Radial Basis Functions,” Engng. Anal. Bound. Elem., vlo.26, pp.205-225, 2002

[5] A.S.M. Wong, Y.C. Hon, et al, "Multizone Decomposition for Simulation of Time-Dependent Problems Using the Multiquadrics Scheme,” Comp. Math. Applic., vol.37, pp.23-43, 1999 